\title{
Heat Transfer Enhancement of Forced Convection in Horizontal Channel with Heated Block due to Oscillation of Incoming Flow
}

\author{
Abdelouahab Bouttout*, Smail Benissaad, Farid Berrahil \\ Laboratoire d'Energétique Appliquée et de Pollution, Département de Génie Mécanique, Université Mentouri- \\ Constantine, Route d'Ain El. Bey, Constantine 25000, Algérie, Algeria
}

\begin{abstract}
The study in question consists to amplify the hydrodynamic and thermal instabilities by imposed pulsation during forced convection of air cooling of nine identical heated blocks simulate electronic components mounted on horizontal channel. The finite volume method has been used to solve the governing equations of unsteady forced convection. This approach uses control volume for velocities that are staggered with respect to those for temperature and pressure. The numerical procedure called SIMPLER is used to handle the pressure-velocity coupling. The results show that the time averaged Nusselt number for each heated block depends on the pulsation frequencies and is always larger than in the steady-state case. The new feature in this work is that we obtained a short band of frequencies which the enhancement of heat transfer of all electronic components is greater than $20 \%$ compared with steady non pulsation flow. In addition, the gain in heat transfer $\mathrm{E}_{\max }$ attainted the maximum value for the central blocks. Our numerical results were compared with other investigations and found to agree well with experimental data.
\end{abstract}

Keywords: Hydrodynamic; Convective Heat Transfer; Oscillation in Fluid Flow

\section{Introduction}

Enhancements of the cooling of electronic equipments by forced convection become an active field of researches. Davalath and Bayazitoglu [1] solved the two-dimensional, conjugate heat transfer problem for laminar flow through an array of three blocks and founded that the local heat flux distribution at the rear surfaces of the obstacles were much smaller than those at the front and top surfaces. In order to enhance heat transfer, numerous methods have been proposed recently, some of these methods are inspired from the natural flow instabilities or initiated by moving turbulator [2]. Kaveh Azar [3] considered that the oscillation of incoming fluid is efficacy method for improving cooling capacity without making major architectural change to the system. Patera and Mikic [4] found that small fluid oscillation at the natural frequency of the hydrodynamic instability increases significantly the amplitude of the instability within a grooved channel, even for Reynolds numbers below the critical value at the onset of self-sustained oscillations, and thus enhances heat

${ }^{*}$ Corresponding author. E-mail: Bouttout@gmail.com

(C) 2014 International Association for Sharing Knowledge and Sustainability

DOI: $10.5383 /$ ijtee.07.01.002

\begin{abstract}
transfer. They called this effect resonant transport enhancement. Jeong Woo Moon et al. [5] examined experimentally the effect of pulsation frequency varied from 10 $\mathrm{Hz}$ to $100 \mathrm{~Hz}$ on the convective heat transfer from protruding heat sources. Their results show that there is a dominant frequency to characterize thermal resonance which is defined as the reciprocal of the travelling time, i.e. $f=U_{c} / L$, where $U_{c}$ is the characteristics velocity over the block array and $L$ the length of periodicity. The turbulent flow field and heat transfer enhancement of mixed convection in a horizontal block-heated channel with rectangular turbulator was investigated by Shiang-Wuu Perng et al. [6].The results indicate that the turbulator mounted in cross-flow above an upstream block can effectively enhance the heat transfer performance of mixed convection in the horizontal channel. The thermal instabilities of natural convection inside a rectangular enclosure with heated block are studied numerically by Bouttout et al. [7]. The critical Rayleigh number values at which transition to unstable flow occurs have been determined for various thermal conductivities of the heat sources and the spacing between them.
\end{abstract}


In the present paper, unsteady pulsation forced convection flow in a two dimensional horizontal channel with nine heated blocks is solved numerically. This model is of practical interest in the cooling of electronic components. The main objective of the paper is to predict the effect of instability amplification by imposed pulsation fluid on the flow and the convective heat transfer rate. Based on the numerical data, the enhancement factor for each block will be evaluated.

\section{Physical Model}

The physical system under consideration, as illustrated in Figure 1, is horizontal channel of height $\mathrm{H}$ and length $\mathrm{L}=40 \times \mathrm{H}$ with nine identical rectangular blocks (electronic components) spaced uniformly on the bottom wall. The height of the heater to height of the channel ratio was set at $\mathrm{h} / \mathrm{H}=0.5$. The length-toheight ratio of the block was fixed at $1 / \mathrm{h}=2$. Inlet and outlet lengths of $\mathrm{L} 1 / \mathrm{H}=11$ and $\mathrm{L} 2 / \mathrm{H}=12$. The top and bottom walls surfaces of the channel are adiabatic. The fluid, at the entrance, is assumed to be at the ambient temperature. At the outlet the velocity and temperature gradients in the axial direction are sets to zero.

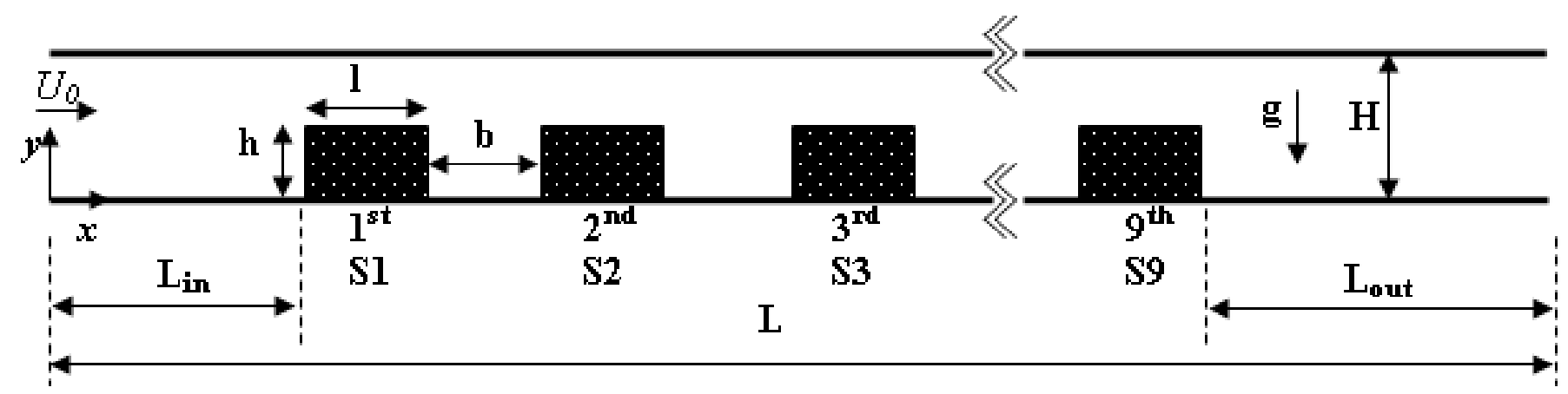

Fig. 1. Schematic configuration of the problem under consideration -Horizontal channel with nine identical heated blocs. We represent only the $1^{\text {st }}$, the $2^{\text {nd }}$, the $3^{\text {rd }}$ and the $9^{\text {th }}$ blocks.

\section{Mathematical Formulation and Boundary Conditions}

Let the flow through the channel be two dimensional, unsteady and laminar. We define the dimensionless variables:

$$
\begin{aligned}
& X=\frac{x}{H}, \quad Y=\frac{y}{H}, \quad U=\frac{u}{u_{0}}, \quad V=\frac{v}{u_{0}} \\
& \tau=\frac{t \cdot u_{0}}{H}, P=\frac{p-p_{0}}{\rho_{0} u_{0}{ }^{2}}, \quad \theta=\frac{T-T_{0}}{T_{s}-T_{0}}, \quad V^{*}=\frac{v}{V_{a}}
\end{aligned}
$$

Where $u$ and $v$ are the velocity component in the $\boldsymbol{x}$ and $\boldsymbol{y}$ direction, respectively. $\mathrm{H}$ is the height of the channel and $\mathrm{t}$ is the time. With neglect of viscous dissipation and Buoyancy force, the governing differential equations in dimensionless form can be expressed as:

$$
\begin{aligned}
& \frac{\partial U}{\partial X}+\frac{\partial V}{\partial Y}=0 \\
& \frac{\partial U}{\partial \tau}+\frac{\partial}{\partial X}(U U)+\frac{\partial}{\partial Y}(U V)=-\frac{\partial P}{\partial X}+\frac{1}{\operatorname{Re}}\left\{\frac{\partial}{\partial X}\left(V^{*} \frac{\partial U}{\partial X}\right)+\frac{\partial}{\partial Y}\left(V^{\prime} \frac{\partial V}{\partial Y}\right)\right\} \\
& \frac{\partial V}{\partial \tau}+\frac{\partial}{\partial X}(U V)+\frac{\partial}{\partial Y}(V V)=-\frac{\partial P}{\partial Y}+\frac{1}{\operatorname{Re}}\left\{\frac{\partial}{\partial X}\left(V^{\cdot} \frac{\partial V}{\partial X}\right)+\frac{\partial}{\partial Y}\left(V^{*} \frac{\partial V}{\partial Y}\right)\right\} \\
& \frac{\partial \theta}{\partial \tau}+\frac{\partial}{\partial X}(U \theta)+\frac{\partial}{\partial Y}(V \theta)=\frac{1}{\operatorname{Re} \cdot \operatorname{Pr}}\left\{\frac{\partial}{\partial X}\left(K^{*} \frac{\partial \theta}{\partial X}\right)+\frac{\partial}{\partial Y}\left(K^{*} \frac{\partial \theta}{\partial Y}\right)\right\}
\end{aligned}
$$

Where $\operatorname{Re}=U_{0} H / v, \quad P r=v / \alpha$ are the Reynolds and Prandl numbers, respectively. In the computational treatment of momentum equations (3) and (4), $v^{*}$ for the block can be set to an infinity large value such as $10^{20}$ to simulate solid blocks. The associated boundary conditions in dimensionless form are:

$$
\begin{array}{llll}
U=0 \text { or } U=1+A \sin (2 \pi S t \tau), V=0, \theta=0 \text { at } & X=0,0 \leq Y \leq 1 & \text { (inlet) } \\
\frac{\partial U}{\partial X}=\frac{\partial V}{\partial X}=\frac{\partial \theta}{\partial X}=0 \text { at } X=40,0 \leq Y \leq 1 & \text { (outlet) } \\
\frac{\partial \theta}{\partial Y}=0 \text { at } Y=0,0 \leq X \leq 40 & \text { (adiabatic wall) } \\
\frac{\partial \theta}{\partial Y}=0 \text { at } Y=1, \quad 0 \leq X \leq 40 & \text { (adiabatic wall) }
\end{array}
$$

Here, the nondimensional frequency parameter called Strouhal number $S t=f H / U_{0}$, is introduced. A is the amplitude oscillation. $\mathrm{A}=0.20$ for all computation.

\section{Results and Discussions}

\subsection{Numerical method and grid sensitivity tests}

The non-dimensional governing equations with the boundary conditions were discretised using a control volume formulation given by Patankar [8]. The components of velocity ( $U$ and $V$ ) are stored at the staggered locations, and scalar quantities (P and $\theta$ ) are stored in the centre of these volume. The SIMPLER algorithm was used to handle the pressure-velocity coupling. For treatment of the convective and diffusion terms in equation (2) and (4), central difference scheme is adopted [8]. Finally, the discretized algebraic equations are solved by the line-byline tri-diagonal matrix algorithm (TDMA). Convergence at a given time step is declared when the maximum change between two consecutive iteration levels fell bellow than $10^{-4}$, for $U, V$ and $\theta$. At this stage, the steady state solution is obtained. The forced convection was implemented in a Visual Fortran program developed by M. Afrid and Zebib [9].

The grid is considered uniform two-dimensional Cartesian grid. In the present work, the grid independence is carried out using three different grid sizes for same computational domain size. Fig. 2 shows the averaged Nusselt number of the block. The number of nodes was chosen to be $2002 \times 52$ after a griddependency test was carried out. 


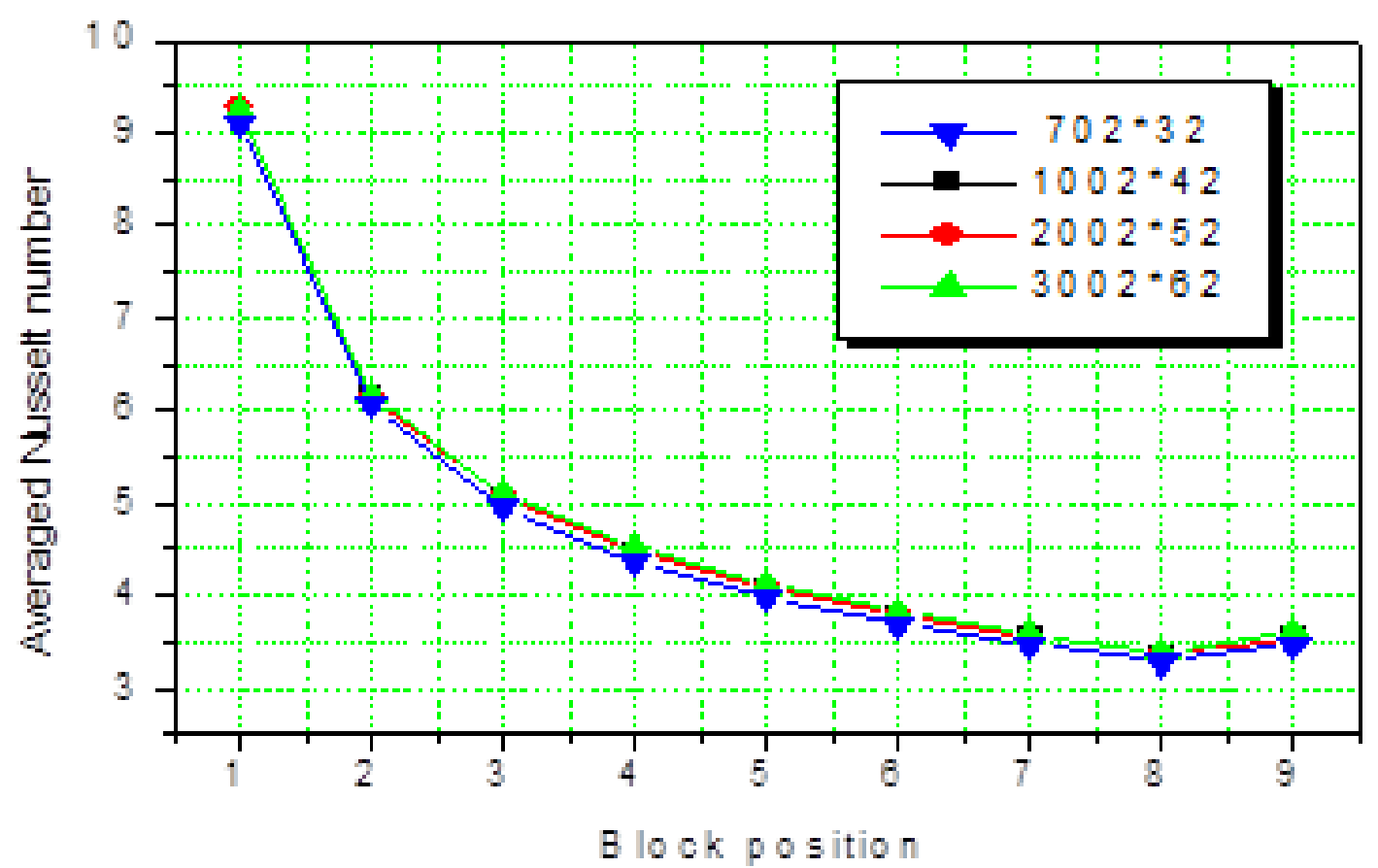

Fig. 2. Averaged Nusselt numbers profiles along the nine heaters for different grid size $\mathbf{R e}=\mathbf{2 0 0}$

\section{2 flow field and isotherms for steady state regime}

Figure 3 displays the distribution of stream function and isotherms for $\mathrm{Re}=100, \mathrm{Re}=200$ and $\mathrm{Re}=300$ (steady non pulsation regime). Due to the obstruction of the first bloc, the streams lines begin to deflect at upstream of bloc corners and the fluid accelerated upward. The fluid in the channel is separated by a shear layer to form weak clockwise vortices between the heated blocks. These secondary flows influence the heat removal rate of the heat sources. Isotherms are clustered in the immediate vicinity of the heated blocks. These lead to thin thermal boundary-layer near the blocks surfaces. Above the heated blocks, the isotherms are parallel to the axial flow. However, in the inter-blocks spacing the isotherms lines are distorted and the thermal transport is characterized by convection mode.

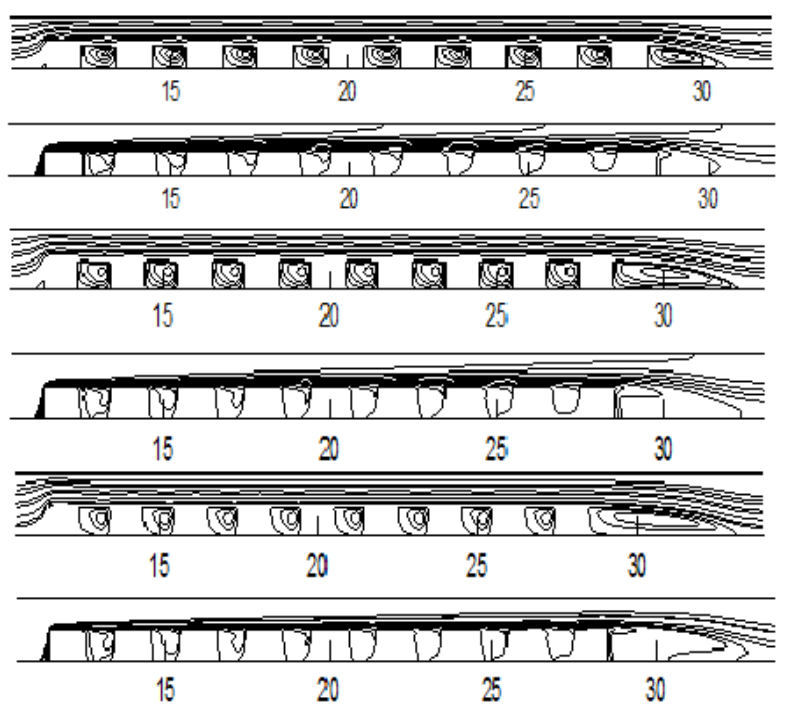

Fig. 3. Streamlines and isotherms for $\operatorname{Re}=100,200$ and 300 .

\subsection{Unsteady pulsation flow}

\subsubsection{Time averaged Nusselt number and enhancement factor}

This section describes temporal variation of the flow for range of sinusoidal forced velocity inlet. The sinusoidal variation of the inlet velocity cased to the problem comes unsteady. With imposing a sinusoidal velocity at the inlet of the channel, after certain time duration, unsteady variation in the fluid flow and temperature field in the channel is observed.

In this case, three flow parameters characterize the pulsatile flow: the net flow Reynolds number based on the channel height, the oscillatory fraction of the flow rate and Strouhal number (dimensionless frequency).

The Nusselt number $\mathrm{Nu}$ at each block is expressed in terms of given heat flux and the measured surface temperature.

Fig.4(a) and 4(b) illustrate the temporal variation of the Nusselt number of nine heated blocks for $\mathrm{Re}=500, \mathrm{Re}=600$ and $\mathrm{A}=0.20$. In the beginning, the difference between steady non pulsation state and pulsation state is small. As the time increase, the effect of velocity pulsation component on heat transfer rate for each block is apparent gradually. Finally, the variation of the heat transfer approaches a periodic state with time $\tau \geq 10$.

To estimate the overall heat transfer rate, the average Nusselt number of the nine heated blocks S1, S2, S3, S4, S5, S6, S7, S8 and $\mathrm{S} 9$ for different Strouhal number (Pulsation frequencies) is plotted in Figs. 5 (a) and 5(b).

It is seen that the instantaneous average Nusselt number of the system is always enhanced in comparison to the steady state case with $\mathrm{Re}=500$ and $\mathrm{Re}=600$. Although the middle of oscillation for all case (all heated block) investigated is greater than the steady state case with $\mathrm{Re}=500, \mathrm{Re}=600$. This must be considered to choose the best Strouhal number for optimising the heat transfer performance. To better realise the optimum resonance band of the Strouhal number, the total heat transfer in a cycle of oscillation of all heated blocs are plotted with 
respect to the Strouhal number in Figs. 5 for Reynolds number $\mathrm{Re}=500$ and $\mathrm{Re}=600$. It is clear that maximum heat transfer gain is remarkably in prescribed value, the range of band frequencies corresponding the maximum heat transfer is about to $0.8-1.40$. The gain in heat transfer $E_{\max }$ in the range of this band is greater than $20 \%$ for all heated block for the case of $\mathrm{Re}=500$ and up to $30 \%$ for the case of $\mathrm{Re}=600$.

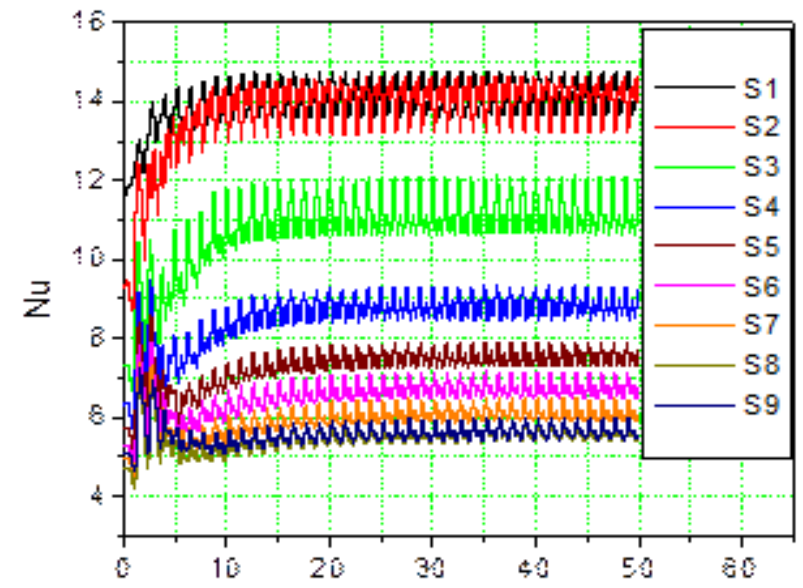

(a) $\bar{i}$

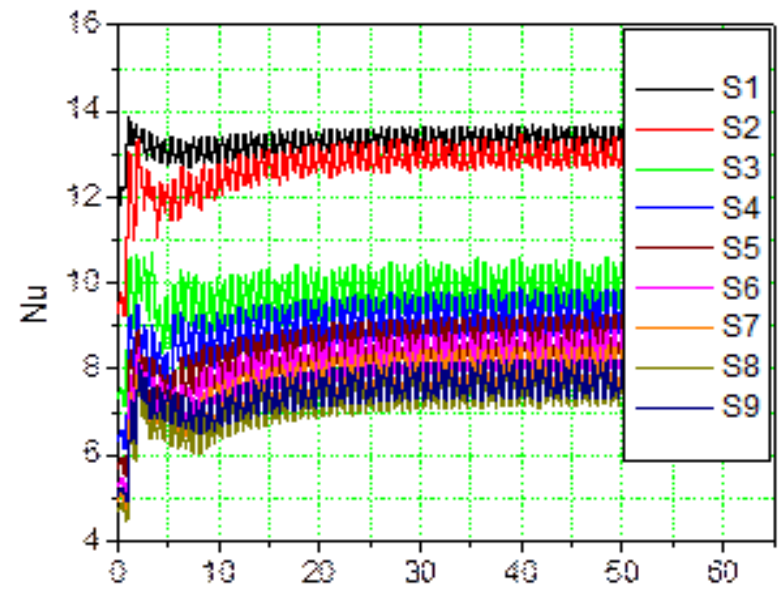

(b) i

Fig.4. Time evolution of Nusselt number of the nine heated block. (a) $\operatorname{Re}=500, \mathrm{St}=0.8$ and $\mathrm{A}=0.2$. (b) $\mathrm{Re}=600, \mathrm{St}=1.4$ and $\mathrm{A}=0.2$

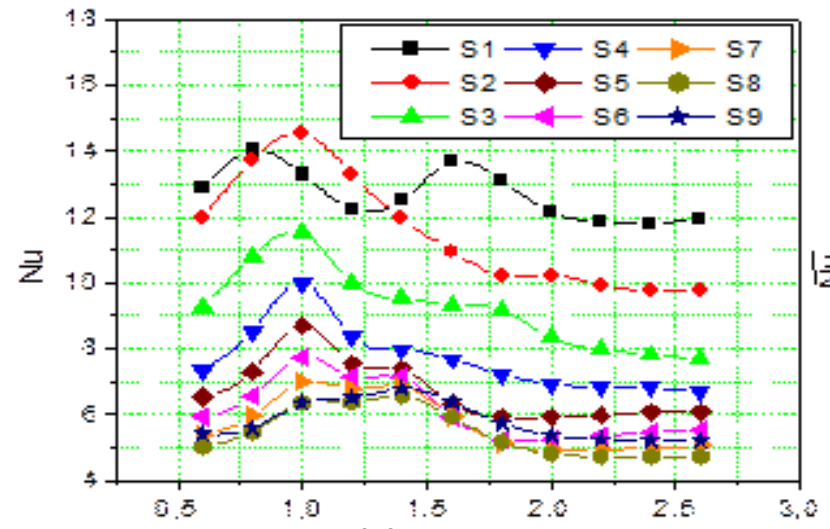

(a) st

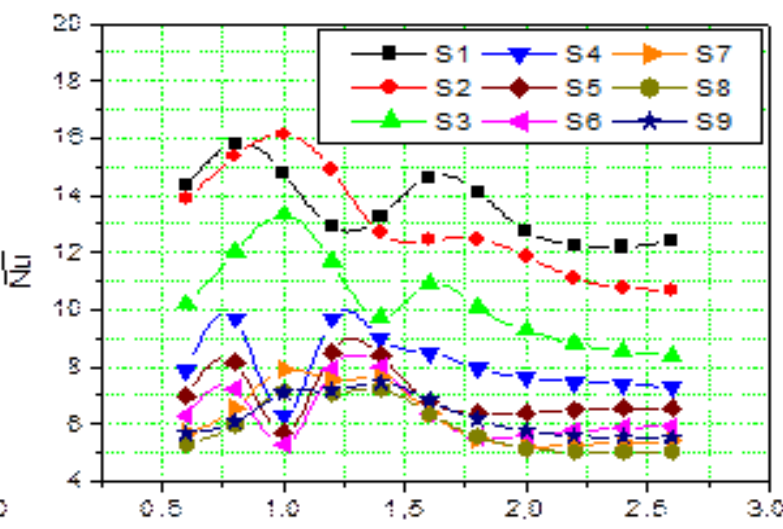

(b) st

Fig. 5. Variation of Nusselt number of the blocs S1, S2, S3, S4, S5, S6, S7, S8 and S9 with Strouhal number. (a) $R e=500$, $A=0.2$, (b) $R e=600, A=0.2$

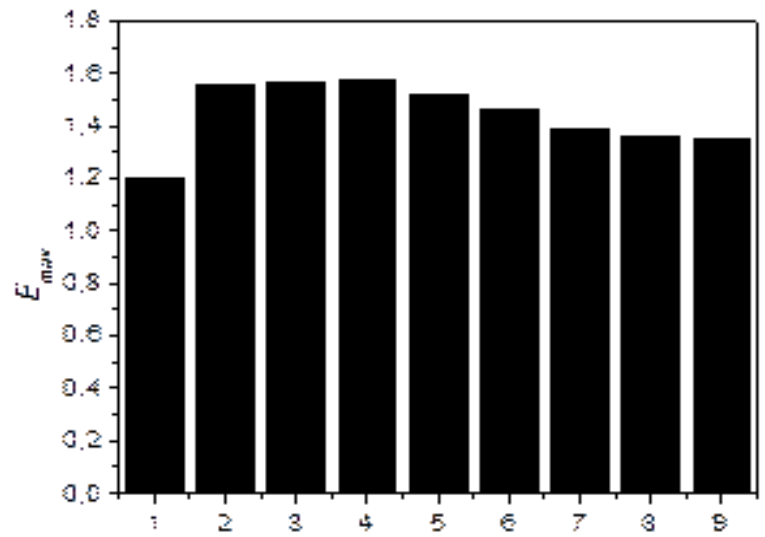

(a) Elock postiso

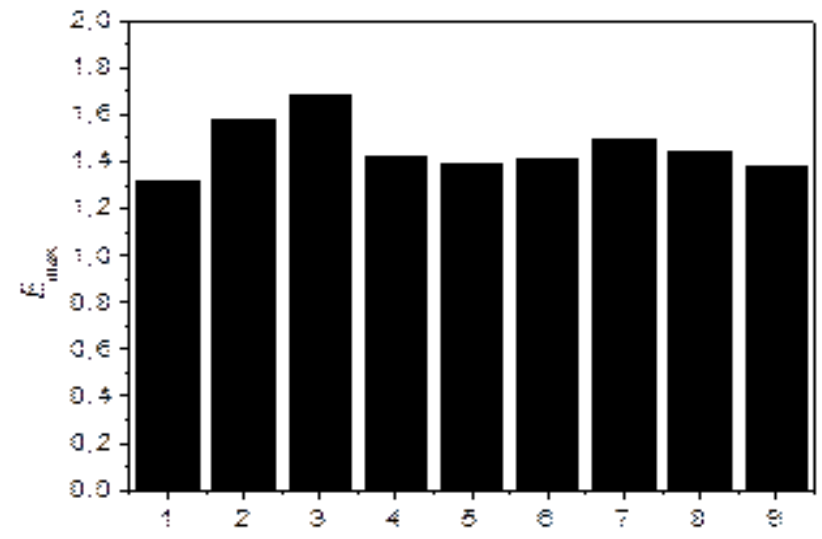

(b) Eiqo pas:i:or:

Fig. 6. Variation of the maximum enhancement heat transfer factor $E_{\max }$ for different blocs positions. (a) $\operatorname{Re}=500, A=0.2$. $(\mathrm{b})$ $\mathrm{Re}=600, \mathrm{~A}=0.2$

\subsubsection{Band width}


In figure 7(a) the band width become wide when Reynolds number approach to critical values. This result indicates that the heat transfer enhancement existed in wider band of frequencies for the Reynolds numbers range [600-700], i.e $\mathrm{Bw} \approx 0.6$; however it is very narrow for the height Reynolds numbers values, i.e $\mathrm{Bw} \approx 0.2$. In this case, two frequencies can contribute of enhancement mechanism; the first is the natural frequency of the system $(S \operatorname{stn}=0.48)$ and the second is the frequency of the pulsation. It should be noted that the band width is the difference between the maximum and minimum Strouhal number value for which the heat transfer gain is maximum i.e, $B_{w}=$ Stmax-Stmin. The band width become small for height Reynolds number, this result indicates that the heat transfer is dominant by self-sustained frequency and the excitation effect is insignificant. For the higher Reynolds numbers values the width of the band become constant i.e, $\mathrm{Bw}=0.2$.

Figure 7(b) shows the enhancement factor versus Strouhal numbers (dimensionless frequencies). It will be noted that there exist a good agreement between numerical code and experimental measurement [5]. The enhancement heat transfer dependent strongly with frequency of the inlet velocity near prescribed value, in this case $\mathrm{St}_{\mathrm{R}}=1.40$.
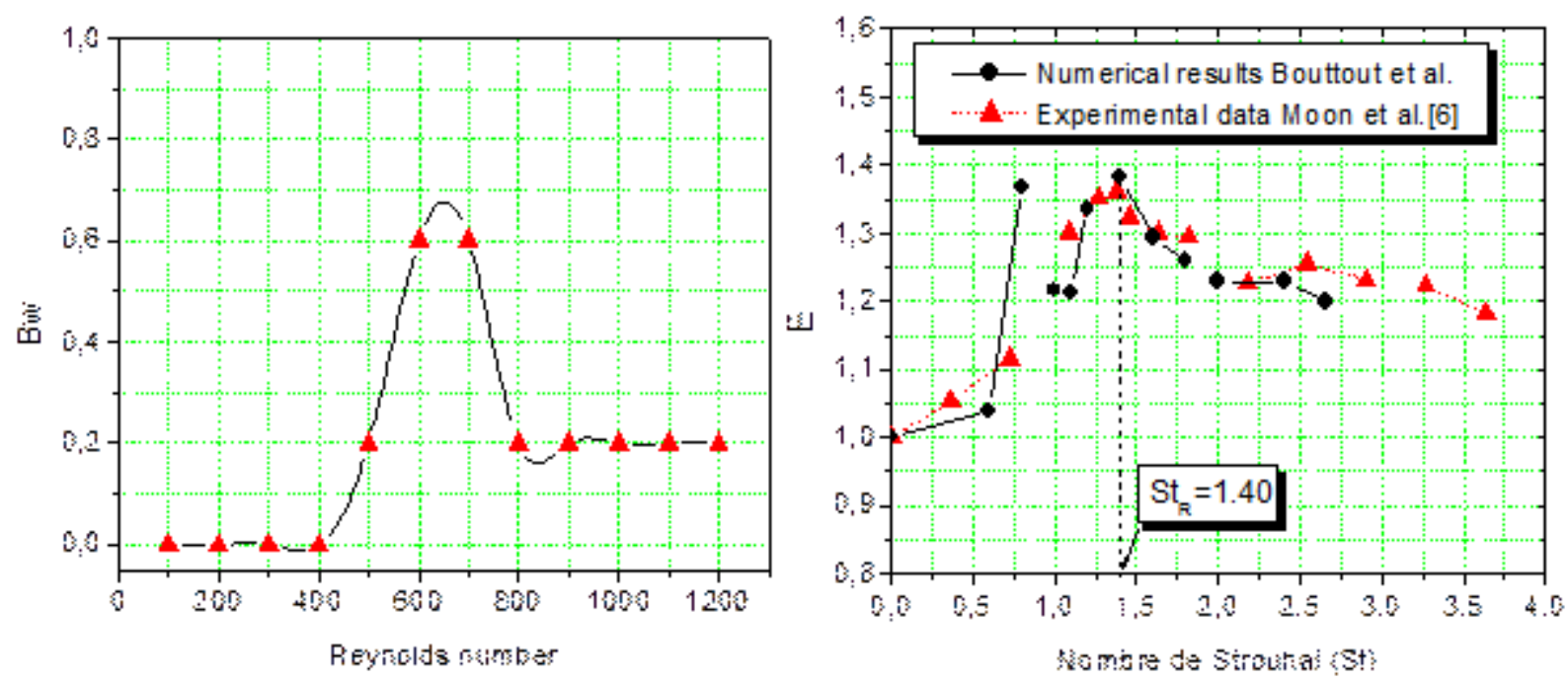

Fig. 7: (a) Variation of the band width with Reynolds number $(\operatorname{Re}=100-1200)$. (b) Enhancement factor versus Strouhal number for the first block, $\mathrm{Re}=700, \mathrm{~A}=\mathbf{0 . 2}$

\section{Conclusion}

The heat transfer characteristics of the heated blocks in the channel are investigated numerically. The results show that the unsteady pulsation flow for the range of Reynolds number $\mathrm{Re}=100-1200$ which characterized by resonance heat transfer phenomena. In this case it is observed that the pulsation flow velocity at the entrance of the channel, after certain time duration, cased to an unsteady variation in the fluid flow and temperature field in the channel. Also as the Strouhal number increase, it takes more number of cycles to reach the periodic state. The heat transfer in this case is enhanced considerably compared to steady non pulsation and the maximum gain can be reached $50 \%$ for the central blocks position. The enhancement factors for all electronic components vary between $25 \%$ and $55 \%$ compared with steady non pulsation flow case. In addition, this band is larger for the upstream and down blocs and become very narrow for the central block. The effect of Reynolds numbers on the band width is very important and it's wide become narrow for the steady state regime and wider for unsteady mod.
Acknowledgement -The authors gratefully acknowledge the financial support of this work (Phd thesis) provided by Algerian Ministry of High Education and Scientific Research.

\section{Nomenclature}

A Oscillating amplitude of axial velocity $\mathrm{A}=U_{p} / U_{0}$

b Inter-block spacing, $\mathrm{m}$

Bw Width of band frequencies

$E_{\text {maxi }}$ Maximum heat transfer factor $[\max (\mathrm{Nu}(\mathrm{st})) / \mathrm{Nu}(\mathrm{St}=0.0)]$

f Dimensional frequency, $\mathrm{Hz}$

g Gravity, m.s ${ }^{-2}$

H,L Height and Length of channel, $m$

h,1 Height and Length of block, m

$\mathrm{P} \quad$ Dimensionless pressure

$\mathrm{p}$ Pressure, $\mathrm{Pa}$

$\mathrm{T}$ Temperature, $\mathrm{k}$ 
$\mathrm{T}$ Time, $\mathrm{s}$

U, V Dimensionless Cartesian velocities

$\mathrm{u}, \mathrm{v}$ Dimensional Cartesian velocities, $\mathrm{m}_{\mathrm{s}} \mathrm{s}^{-1}$

X, Y Dimensionless Cartesian coordinates

P Dimensionless pressure

$\mathrm{p}$ Pressure, $\mathrm{Pa}$

Greek Symbols

$\alpha \quad$ Thermal diffusivity, $\mathrm{m}^{2} \cdot \mathrm{s}^{-1}$

$\rho$ Mass density, $\mathrm{kg} / \mathrm{m}^{3} \mathrm{v}$

$v$ Kinematic viscosity, $\mathrm{m}^{2} \cdot \mathrm{s}^{-1}$

$\beta$ Thermal expansion coefficient, $\mathrm{k}^{-1}$

$\theta$ Dimensionless temperature

$\tau$ Dimensionless time

\section{Subscripts}

a Air

in Inlet

out Outlet

s Solid

Exponents

* Non dimensional value

Dimensionless Numbers

$\overline{N u}$ Averaged Nusselt number $[h H / k]$

Pr Prandtl number $\quad[v / \alpha]$

Re Reynolds number $\quad\left[u_{0} H / v\right]$

St Strouhal number $\quad\left[f H / u_{0}\right]$

\section{References}

[1] J. Davalath, Bayazitoglu, Forced convection cooling across rectangular blocks, Journal of heat transfer 109 (1987) 321-328.

[2] S. Y. Kim, B. kang, Forced convection heat transfer from two heated blocks in pulsating channel flow. International Journal of Heat and Mass Transfer, Vol.41, N³, pp.625-634, 1998.

[3] Kaveh Azar, Enhanced cooling of electronic components by flow oscillation, Journal of thermophysics and heat transfer Vol. 6, N. 4, OCT.Dec.1992.

[4] A.T. Patera, B.B. Mikic, Exploiting hydrodynamic instabilities, resonant heat transfer enhancement, Int. J. Heat Mass Transfer 29 (1986) 1127-1138.

[5] J.W. Moon, S.Y. Kim, H.H. Cho, Frequencydependent heat transfer enhancement from rectangular heated block array in a pulsating channel flow. International Journal of Heat and Mass Transfer, Vol.48, pp 4904-4913, 2005.

[6] Shiang-Wuu Perng a, Horng-Wen Wu, Numerical investigation of mixed convective heat transfer for unsteady turbulent flow over heated blocks in a horizontal channel, International Journal of Thermal Sciences 47 (2008) 620-632.

[7] A. Bouttout, R. Bessaih, Steady and oscillatory natural convection air cooling of protruding thermal sources mounted in rectangular enclosure, Journal of energy, heat and mass transfer. Vol 30, pp251-271, 2008.

[8] S.V Patankar, Numerical heat transfer and fluid flow,Hemisphere. Washington, DC 1980.

[9] M. Afrid, Three dimensional natural convection. Phd Thesis, Rutgers University, 1990, New Jersey. USA. 